Proyecciones

Vol. 15, $\mathrm{N}^{o} 2$, pp. 153-168, December 1996

Universidad Católica del Norte

Antofagasta - Chile

\title{
UNE APPLICATION DU CALCUL "UMBRAL" NON CLASSIQUE EN TOPOLOGIE ALGĖBRIQUE
}

\author{
Faysal Lamrini \\ Universit Sidi Mohammed Benabdallah, Marruecos
}

\begin{abstract}
En utilisant les techniques du calcul "umbral" non classiques défini par le bigroupe formel élémentaire du $1^{\text {er }}$ type (b.f.c.1) sur $E_{*}(p t) \otimes \mathbf{Q}$, on calcule l'image de $E_{*}\left(\mathbf{H P}^{\infty}\right)$ par l'homomorphisme d'Hurewicz $E \rightarrow E \wedge \mathbf{H Q}$, o $E$ est un spectre-anneau dfinissant une thoric cohomologique $\mathbf{C}$-orientée spéciale.
\end{abstract}




\section{Introduction}

\subsection{Théorie cohomologique}

On rappelle (cf [10] par xemple) qu'un spectre est une famille de (WWcomplexes $E_{n}$ tels que pour tout n, la suspension de $E_{n}$ est un souscomplexe de $E_{n+1}$. On dira que $E$ est un spectre-anneau s'il existe deux applications de spectres $E \wedge E \stackrel{\nu}{\rightarrow} E$ et $S^{0} \stackrel{\iota}{\rightarrow} E$ (où $S^{0}$ est le spectre des sphères) faisant commuter un certain nombre de diagrammes.

Tout spectre $E$ définit sur la catégorie des C.W-complexes une théorie homologique

$$
E_{n}(X)=\lim _{k \rightarrow \infty}\left[S^{n+k}, E_{k} \wedge X\right]
$$

et une théorie cohomologique

$$
E^{n}(X)=\lim _{k \rightarrow \infty}\left[S^{k} \wedge X, E_{n+k}\right]
$$

Ainsi, par exemple la cohomologie ordinaire à coefficients dans un groupe $G, H(-, G)$, est donnée par le spectre de Mac-Lane $\mathcal{H} G$.

Maintenant on dira que le spectre $E$ définit une théorie cohomologique $\mathbf{C}$ orientếsi pour tout fibré vectoriel complexe $\xi \downarrow B$ de rang n et de base un $\mathrm{CW}$-complexe $\mathrm{B}$ il existe une classe de cohomologie $u \in \tilde{E}^{2 n}(\tau \xi)$ dont la restriction à $S^{2 n}$ soit un gnrateur de $\tilde{E}^{*}\left(S^{2 n}\right)$ en tant que $E^{*}(p t)$-module, où $\tau \xi$ est l'espace de Thom de $\xi$. On a alors un isomorphisme de Thom

$$
\phi_{\xi}: E^{*}(B) \rightarrow \tilde{E}^{*}(\tau \xi),
$$

et des classes de Chern pour la théorie $E$.

En particulier $E^{*}\left(\mathbf{C P}^{\infty}\right)=E^{*}(p t)[[u]]$ où $u \in E^{2}\left(\mathbf{C P}^{\infty}\right)$ est la classe de Thom du fibré, en droites complexes, universel $\xi \backslash \mathbf{C P}^{\infty}$.

Puisque $\xi \otimes \xi$ est aussi un fibré en droites complexes sur $\mathbf{C P}^{\infty} \times \mathbf{C P}^{\infty}$, sa classe de Thom est un élément $F(u \otimes 1,1 \otimes u)$ de $E^{*}\left(\mathbf{C P}^{\infty}{ }^{\infty} \mathbf{C P}^{\infty}\right) \cong$ $E^{*}[[u \otimes 1,1 \otimes u]]$ où $F \in E^{*}(p t)[[X, Y]]$ est appelé groupe formel de la théorie cohomologique $E$.

Le groupe formel se définit de façon générale comme suit (cf. [3]).

Définition 1.1.1. On appelle groupe formel à coefficients dans un anneau sans torsion $R$, toute série formelle $F$ de $R[[X, Y]]$ vérifiant les propriétés suivantes :

1. $F(X, Y)=F(Y, X)$ 
2. $F(X, 0)=X$

3. $F(F(X, Y), Z)=F(X, F(Y, Z))$

le logarithme de $F$ est l'élement $g$ de $R \otimes \mathbf{Q}[[\mathbf{X}]]$ tel que :

1. $g(X)=X+\left(X^{2}\right)$

2. $g(F(X, Y))=g(X)+g(Y)$

Exemples 1.1.2.

1) La cohomologie ordinaire est $\mathbf{C}$-orientée de groupe formel $F_{a}(X, Y)=$ $X+Y$ (le groupe formel additif).

2) La K-théorie est aussi une thorie cohomologique C-orientée de groupe formel $F_{m}(X, Y)=X+Y-\beta X Y$ (groupe formel multiplicatif) où $\beta \in$ $K^{-2}(p t)$ est l'lment de priodicit de Bott.

3) Le cobordisme complexe est universel pour les théories cohomologiques C-orientes, son groupe formel est

$$
F^{U}(X, Y)=X+Y+\sum_{i+j=n} \frac{\left[H_{i, j}\right]}{\mathbf{C P}(\mathbf{X}) \mathbf{C P}(\mathbf{Y})} X^{\imath} Y^{\jmath}
$$

où les $\left[H_{i, j}\right]$ sont les sous-variétés de Milnor, $\mathbf{C P}(\mathbf{X}):=\sum_{\mathbf{n}}\left[\mathbf{C P}^{\mathbf{n}}\right] \mathbf{X}^{\mathbf{n}}$.

\subsection{Calcul "umbral"}

Nous allons donner juste une idée sur le sujet, pour uneátude en profondeur de celui-ci on renvoie à [8] et [6] entre autres.

Soit $\mathbf{R}$ une $\mathbf{Q}$-algèbre, on appelle algebre "umbrale" l'algebre $R[[X]]^{*}=$ $H o m_{R}(R[[X]], R)$ dont le produit est l'application duale du coproduit $\delta$ de $R[[X]]$ défini par,

$$
\begin{aligned}
\delta: \quad R[[X]] & \rightarrow R[[X]] \otimes R[[X]]=R[[X, Y]] \\
P(X) & \rightarrow P(X+Y)
\end{aligned}
$$

Les éléments de $R[[X]]^{*}$ sont appelés fonctionnelles.

On note $\mathcal{A}$ la fonctionnelle canonique définie $\operatorname{par}\left\langle\mathcal{A}, X^{n}\right\rangle=\delta_{1, n}$. Le système $\left\{\mathcal{A}^{n}\right\}_{n \in \mathrm{N}}$ constitue une base topologique de $R[[X]]^{*}$, relativement à la topologie I-adique ( I est l'ideal $X R[[X]]$ ), duale de la base polynômiale $\left\{X^{n}\right\}_{n \in \mathbb{N}}$. Toute fonctionnelle $\mathcal{M}$ s'crit

$$
\mathcal{M}=\sum_{n=0}^{\infty}\left\langle\mathcal{M}, X^{n}\right\rangle \frac{\mathcal{A}^{n}}{n !}
$$


La série formelle $\varphi_{\mathcal{M}}(X)=\sum_{n \leqslant 0}\left\langle\mathcal{M}, X^{n}\right\rangle \frac{X^{n}}{n !}$ est caracterstique de $\mathcal{M}$ et de ce fait appelée indicatrice de $\mathcal{M}$.

Par extension on montre que les puissances de toute $\delta$-fonctionnelle $\mathcal{L}$ (i.e. $\langle\mathcal{L}, 1\rangle=0$ et $\langle\mathcal{L}, X\rangle=1$ ) constituent une base topologique de $R[[X]]^{*}$; son systme dual $\left\{p_{n}\right\}_{n \in \mathbf{N}}$ est une base polynomiale de $R[[X]]$ telle que

1. $p_{0}=1$

2. degré de $p_{n}=n$

3. $\left(\delta p_{n}\right)(X)=\sum_{i=0}^{i=n} C_{i}^{n} p_{i}(X) p_{n-i}(Y)$

appelée la base binomiale de $R[[X]]$ associée à $\mathcal{L}$.

Notons enfin que si $\mathcal{M}$ et $\mathcal{N}$ sont deux fonctionnelles, leur composition $\mathcal{M a N}$ est par définition la fonctionnelle d'indicatrice $\varphi_{\mathcal{M}} 0 \varphi_{\mathcal{N}}$. Ainsi le sous-ensemble des $\delta$-fonctionnelles est un groupe non commutatif pour la composition. Si $\mathcal{L}$ est une $\delta$-fonctionnelle, son inverse $\mathcal{L}^{-1}$ est appelée conjuguée de $\mathcal{L}$ d'indicatrice $\varphi_{\mathcal{L}^{-1}}=\varphi_{\mathcal{L}}^{-1}$. La base binomiale associée à $\mathcal{L}^{-1}$ est dite conjugue de $\mathcal{L}$.

\subsection{Lien avec la topologie algèbrique}

Soit $E$ un spectre-anneau définissant une théorie cohomologique $\mathbf{C}$-orientée, de groupe formel $F$ et de logarithme $g . E_{*}\left(\mathbf{C P}^{\infty}\right)$ est un $E_{*}(p t)$-module libre de base $\left\{b_{i}^{E}\right\}_{i \in \mathbf{N}} \cdot b_{i}^{E} \in E_{2 i}\left(\mathbf{C P}^{\infty}\right)$ est défini par $\left\langle u^{3}, b_{i}^{E}\right\rangle=\delta_{i, j}$ où $\langle$, est le produit de Kronecker. En plus la structure de H-espace sur $\mathbf{C P}^{\infty}$ induit le produit de Pontrjagin sur $E_{*}\left(\mathbf{C P}^{\infty}\right)$ défini par

$$
b_{i}^{E} b_{j}^{E}=\sum_{k}\left\langle F(u \otimes 1,1 \otimes u)^{k}, b_{i}^{E} \otimes b_{j}^{E}\right\rangle b_{k}^{E}
$$

En particulier pour la cohomologie ordinaire, $F_{a}(X, Y)=X+Y$, le produit de Pontrjagin sur $H_{*}\left(\mathbf{C} \mathbf{P}^{\infty}, \mathbf{Z}\right)$ est donn par $b_{i}^{H} b_{j}^{H}=C_{i}^{n+j} b_{i+j}^{H}$, par conséquent $H_{*}\left(\mathbf{C P}^{\infty}, \mathbf{Q}\right)=\mathbf{Q}\left[\mathbf{b}_{\mathbf{1}}^{\mathbf{H}}\right]$. Soit $\mathcal{H} \mathbf{Q}$ le spectre de Mac:Lane. L'application $\tau: E \simeq S^{0} \wedge E \stackrel{{ }^{\wedge} 1_{E}}{\longrightarrow} \mathcal{H} \mathbf{Q} \wedge \mathbf{E}$ induit l'homomrphisme d'Hurewicz $\tau_{*}: E_{*}\left(\mathbf{C P}^{\infty}\right) \rightarrow E_{*}(p t) \otimes \mathbf{Q}\left[\mathbf{b}_{\mathbf{1}}^{\mathbf{H}}\right]$. Les techniques du calcul "umbral" classique permettent de donner une interprétation intéressante de $\tau_{*}\left(b_{n}^{E}\right)$; en effet on a le résultat suivant. 
Théorème([7]). $\tau_{*}\left(b_{n}^{E}\right)=\frac{1}{n !} p_{n}\left(b_{1}^{H}\right)$ où $p_{n}$ est le $n^{\text {ème }}$ polynôme de la suite binomiale conjuguée de la $\delta$-fonctionnelle sur $E_{*}(p t) \otimes \mathbf{Q}\left[\mathbf{b}_{1}^{\mathbf{H}}\right]$ d'indicatrice $g$.

On se propose, dans ce travail, de généraliser le théorème ci-dessus au cas du projectif quaternionique, sous la condition que la théorie cohomologique définie par $E$ soit spéciale (i.e. $g$ est une série impaire) et ce en utilisant les techniques du calcul "umbral" non classique (i.e. le produit de l'algebre "umbrale" est l'application duale du coproduit sur $E^{*}(p t) \otimes \mathbf{Q}[[\mathbf{X}]]$ défini par le bigroupe formel associé à la théorie cohomologique $E$ ).

\section{Calcul "umbral" non classique}

Nous entendons par calcul "umbral" classique, la structure d'algbre sur $R[[X]]^{*}$ obtenue à partir du groupe formel additif, c-à-d. à partir du coproduit $\delta: \varphi(X) \rightarrow \varphi(X+Y)=\varphi\left(F_{a}(X, Y)\right)$ où $F_{a}$ est le groupe formel additif. On peut développer une théorie semblable en remplaçant le groupe formel additif par un groupe formel quelconque, c'est le calcul "umbral" non classique. Dans ce qui suit nous nous intéresserons au cas, plus général encore, du calcul "umbral" introduit par un bigroupe formel.

\subsection{Bigroupe formel}

Pour plus de détails, à ce sujet, on pourra consulter [1].

Définition 2.1.1. Soit $R$ un anneau commutatif unitaire.

On appelle bigroupe formel sur $\mathrm{R}$, noté $\mathcal{F}$, l'équation du second ordre

$$
\mathcal{F}^{2}-\theta_{1} \mathcal{F}+\theta_{2}=0
$$

où $\theta_{1}, \theta_{2} \in R[[X, Y]]$ vérifient les propriétés suivantes :

a) $\theta_{i}(X, Y)=\theta_{i}(Y, X), \quad i=1,2$

b.) $\theta_{i}(X, 0)=C_{i}^{2} X^{i}, \quad i=1,2$

c) $\mathcal{F}(\mathcal{F}(X, Y), Z)=\mathcal{F}(X, \mathcal{F}(Y, Z))$,

avec la convention suivante: en écrivant symboliquement $\theta_{1}(X, Y)=A+B$ et $\theta_{2}(X, Y)=A \cdot B$ alors $\mathcal{F}(\mathcal{F}(X, Y), Z)$ est l'équation

$$
\left(\mathcal{F}^{2}-\theta_{1}(A, Z) \mathcal{F}+\theta_{2}(A, Z)\right)\left(\mathcal{F}^{2}-\theta_{1}(B, Z) \mathcal{F}+\theta_{2}(B, Z)\right)=0 .
$$




\section{Remarques 2.1.2:}

a) Dans l'équation $\mathcal{F}(\mathcal{F}(X, Y), Z)$, étant donné que les coefficients des puissances de $\mathcal{F}$ sont symétriques en $A, B$ ce sont bien des séries formelles en $X, Y, Z$.

b) Tout groupe formel $F$ est trivialement un bigroupe formel, $(\mathcal{F}-F)^{2}=0$.

c) $\theta_{1}$ et $\theta_{2}$ sont de la forme :

i) $\theta_{1}(X)=2 X+2 Y+\sum_{\substack{i+3>2 \\ i j \neq 0}} a_{i j} X^{i} Y^{\jmath}$

ii) $\theta_{2}(X)=X^{2}+\gamma X Y+Y^{2}+\sum_{\substack{i+j \zeta_{3} \\ i j \neq 0}} b_{i j} X^{i} Y^{\jmath}$

Définition 2.1.3. Le bigroupe formel $\mathcal{F}$ est dit

principal

$d u 1^{\text {er }}$ type si $\gamma=-2$

du $2^{\text {ème }}$ type si $\gamma=2$

élémentaire

du $1^{\text {er }}$ type si $\theta_{1}(X, Y)=2 X+2 Y$ et $\theta_{2}(X, Y)=(X-Y)^{2}$

du $2^{\text {ème }}$ type si $\theta_{1}(X, Y)=2 X+2 Y$ et $\theta_{2}(X, Y)=(X+Y)^{2}$

N.B. On notera dans la suite $\mathcal{F}_{a}$ le bigroupe formel élémentaire du $1^{\text {er }}$-type (b.f.e.1.) $\mathcal{F}_{a}^{2}-2(X+Y) \mathcal{F}_{a}+(X-Y)^{2}=0$.

Rappelons qu'un homomorphisme de groupes formels de $F$ vers $G$ (à coefficients dans un anneau $R$ ) est par définition toute série formelle $\varphi$ de $R[[X]]$ sans terme constant telle que $\varphi(F(X, Y))=G(\varphi(X):, \varphi(Y))$. Par extension on a :

Définition 2.1.4. Si $\mathcal{F}^{2}-\theta_{1} \mathcal{F}+\theta_{2}=0$ et $\mathcal{G}^{2}-\omega_{1} \mathcal{G}+\omega_{2}=0$ sont deux bigroupes formels sur un anneau $R$, on appelle homomorphisme de $\mathcal{F} \rightarrow \mathcal{G}$ toute série formelle $\psi \in R[[X]]$ sans terme constant telle que

$$
\begin{aligned}
& \omega_{1}(\psi(X), \psi(Y))=\psi(A)+\psi(B) \\
& \omega_{2}(\psi(X), \psi(Y))=\psi(A) \cdot \psi(B) \quad(c f . \text { Définition 2.1.1) }
\end{aligned}
$$

$\psi$ est dit un isomorphisme si $\psi^{\prime}(0)$ est inversible dans $R$. C'est un isomorphisme strict si en plus $\psi^{\prime}(0)=1$. 


\subsection{Calcul "umbral" défini par un bigroupe formel}

Dans toute la suite on supposera que $R$ est un anneau commutatif unitaire intégre et sans torsion tel que 2 soit inversible.

Définition 2.2.1. Un bigroupe formel $\mathcal{F}^{2}-\theta_{1} \mathcal{F}+\theta_{2}=0$ définit un coproduit sur $R[[X]]$ par

$$
\begin{aligned}
\Delta_{\mathcal{F}}: R[[X]] & \rightarrow R[[X, Y]] \\
\varphi(X) & \mapsto \frac{1}{2}(\varphi(A)+\varphi(B))
\end{aligned}
$$

où $A, B$ sont des variables intermediaires données comme précédemment par

$\theta_{1}(X)=A+B$ et $\theta_{2}(X)=A \cdot B$.

Par dualité on a un produit $\Delta_{\mathcal{F}}^{*}$ sur $R[[X]]^{*}$, l'ensemble des formes linéaires continues, définissant ainsi le calcul "umbral" non classique.

Remarque 2.2.2. : $\Delta_{\mathcal{F}}$ est détérminé par la formule suivante,

$$
\begin{aligned}
\Delta_{\mathcal{F}}\left(\frac{1}{1-X t}\right) & =\sum_{n \geq 0}\left(\Delta_{\mathcal{F}} X^{n}\right) t^{n}=\frac{1}{2}\left(\frac{1}{1-A t}+\frac{1}{1-B t}\right) \\
& =\frac{1-\frac{1}{2} \theta_{1}(X, Y) t}{1-\theta_{1}(X, Y) t+\theta_{2}(X, Y) t^{2}} .
\end{aligned}
$$

On considère $d_{n}$ les opérateurs invariants de $\Delta_{\mathcal{F}}$ i.e. $d_{n}: R[[X]] \rightarrow R[[X]]$ sont des applications $R$-linéaires telles que le diagramme suivant soit commutatif

$$
\begin{array}{ccc}
R[[X]] & \stackrel{d_{n}}{\longrightarrow} & R[[X]] \\
\Delta_{\mathcal{F} \downarrow} & & \downarrow \Delta \mathcal{F} \\
R[[X, Y]] & \stackrel{d_{n} \otimes 1}{\longrightarrow} & R[[X, Y]] \\
& \stackrel{\longrightarrow \otimes d_{n}}{\longrightarrow} &
\end{array}
$$

définis par

$$
\Delta_{\mathcal{F}} \varphi(X, Y)=\sum_{n \in \mathbf{N}}\left(d_{n} \varphi\right)(X) Y^{n} \quad \forall \varphi \in R[[X]]
$$

Alors,

$$
d_{1}=\frac{1}{2} \varphi_{1}(X) \frac{d}{d X}+\frac{1}{8} \varphi_{2}(X) \frac{d^{2}}{d X^{2}}
$$


où

$\varphi_{1}(X)=\frac{\partial \theta_{1}}{\partial Y}(X, 0)$ et $\varphi_{2}(X)=\frac{\partial}{\partial Y}\left(\theta_{1}^{2}-4 \theta_{2}\right)(X, 0)$.

Proposition 2.2.3.([1]). Soit $\mathcal{F}$ un bigroupe formel sur $R$ induisant une structure d'algèbre ("umbrale") sur $R[[X]]^{*}$ et $\mathcal{D}$ la R-algèbre des

opérateurs invariants sur $R[[X]]$, (ie. $\mathcal{D}=\left\{u \in \operatorname{Hom}_{R}(R[[X]], R[[X]]) / \Delta_{\mathcal{F}}\right.$ o $\left.\left.u=(u @ 1) \circ \Delta_{\mathcal{F}}=\Delta_{\mathcal{F}} \circ(u \otimes 1)\right\}\right)$, alors.

i) $\mathcal{D}$ est une $R$-algèbre libre de base $\left\{d_{n}\right\}_{n \in \mathrm{N}}$

ii) Si $\mathcal{F}$ est principal alors $\mathcal{D} \otimes \mathbf{Q} \simeq \mathbf{R} \otimes \mathbf{Q}\left[\mathbf{d}_{\mathbf{1}}\right]$.

Donnons à présent le théorème de classification des bigroupes formels principaux (cf.[1])

Théorème 2.2.4. Soient $\mathcal{F}^{2}-\theta_{1} \mathcal{F}+\theta_{2}=0$ un bigroupe formel principal sur $R, d_{1}=\frac{1}{2} \varphi_{1}(X) \frac{d}{d X}+\frac{1}{8} \varphi_{2}(X) \frac{d^{2}}{d X^{2}}$ son premier opérateur invariant, alors :

a) Si $\mathcal{F}$ est du $1^{\text {er }}$ type, la série formelle $\psi(X)$ définie par

$$
\psi(X)=\left(\int_{0}^{\sqrt{X}} \frac{d t}{\sqrt{\varphi_{2}\left(t^{2}\right) / 16 t^{2}}}\right)^{2}
$$

est un isomorphisme strict entre $\mathcal{F}$ et le b.f.e.1. $\mathcal{F}_{a}$ sur $R$, appelé logarithme de $\mathcal{F}$.

b) Si $\mathcal{F}$ est du $2^{\text {ème }}$ type, alors $\frac{\theta_{1}}{2}$ est un groupe formel sur $R$ de logarithme

$$
g(X)=\int_{0}^{X} \frac{d t}{\varphi_{1}(t)}
$$

On peut envisager aisemment la réciproque,

Proposition 2.2.5. Pour tout élément $\psi$ de $R[[X]]$ tel que $\psi(X)=$ $X+\left(X^{2}\right)$, il existe sur $R \otimes \mathbf{Q}[[\mathbf{X}]]$ un bigroupe formel principal du $1^{\text {er }}$ type de logarithme $\psi$. 
Démonstration : $\$$ i un tel higronpe existe, notons-le $\mathcal{F}$, $\mathcal{H}^{\prime}$ sera un inticrplishus itriet

$$
\because H^{2}-H_{1} f+\theta_{2} \longrightarrow F_{1}^{2}-2(X+Y) F_{a}+(X-Y)^{2}
$$

, .1 .1$.

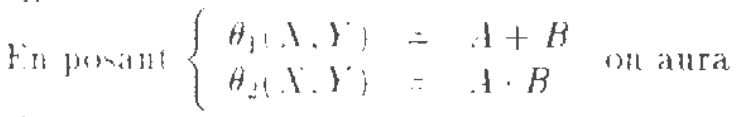

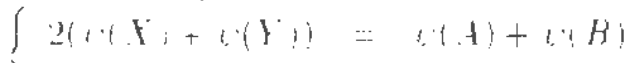

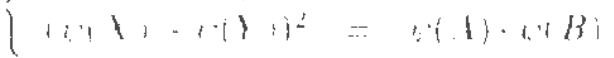

i.

$$
\left.\left.6^{2}-2(1.4)+4(Y)\right) 6+(4)-6(Y)\right)^{2}=0
$$

inilic

$$
1 \cdot(A)-\left(\sqrt{u(X)}+\sqrt{(+Y))^{2}}\right.
$$

11

$$
\psi(B)=(\sqrt{4(X)}-\sqrt{w(Y)})^{2}
$$

Noist

$$
\begin{aligned}
& \left.\left.H_{1}+.\right\}\right)=A+B=b^{-1}\left([\sqrt{(Y X)}+\sqrt{(y)}]^{2}\right)+w^{-1}\left([\sqrt{\psi(X)}-\sqrt{\psi(Y)}]^{2}\right)
\end{aligned}
$$

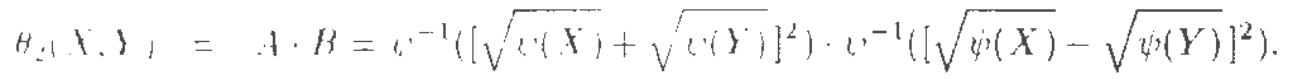

Remarque 2.2.6. Do facost gerériale un sait, d"après [A], que si $\Delta X^{n}=$

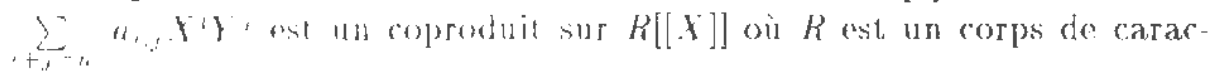

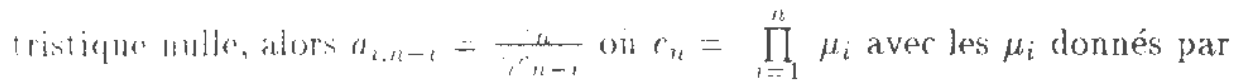

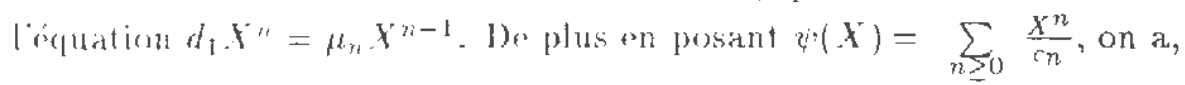
a) $\Delta i^{2}=y_{1}^{\prime}: \dot{w}$

b) Pour toute $h$-fonctionnelle $L$ d"indicatrice $\varphi$ (i.e. $L=\varphi(\mathcal{A})=$ $\left.\sum_{n \leq 0}\left\langle L, X^{n}\right\rangle \frac{A^{n}}{c_{n}}\right)$ on a $\psi(X \varphi(t))=\sum_{n \geq 0} \frac{\eta_{n}(X)}{c_{n}} t^{n}$ oì $\left\{q_{n}\right\}$ est la suite binomiale

(i.e. $\Delta q_{n}=\sum_{i+j=n} a_{i, j} q_{i} q_{j}$ ) conjuguée de $L$ pour le calcul "umbral" défini par $\Delta$. 
Exemple 2.2.7 : le coproduit défini par $\mathcal{F}_{a}$ est

$$
\Delta_{a} X^{n}=\sum_{i=0}^{n} C_{2 i}^{2 n} X^{i} Y^{n-j}
$$

En effet d'après (1) $d_{1}=\frac{d}{d X}+2 X \frac{d^{2}}{d X^{2}} ;$ lonc $\forall n \geq 1, d_{1} X^{n}=n(2 n-1) X^{n-1}$ et on a $\mu_{n}=n(2 n-1)$, d'où $c_{n}=\prod_{i=1}^{i=n} \mu_{i}=\prod_{i=1}^{i=n} i(2 i-1)=\frac{(2 n) !}{2^{n}}$. Donc $a_{i, n-i}=\frac{(2 n) !}{(2 i) !(2(n-i)) !}=C_{2 i}^{2 n}$

\section{Application à la topologie algèbrique}

\subsection{Bigroupe formel associ é une théorie cohomologique C-orientée}

Soit $h^{*}$ une théorie cohomologique C-orientée, de groupe formel $F$ et de logarithme $g ; c_{i}^{h}$ ses classes de Chern. A $h^{*}$ est associé un bigroupe formel principal du $1^{\text {er }}$ type à coefficients dans $h^{*}(p t)$ donné par l'équation, $\mathcal{F}^{2}-$ $\theta_{1} \mathcal{F}+\theta_{2}=0$ où les séries formelles $\theta_{1}$ et $\theta_{2}$ sont définies de la manière suivante :

notons $\zeta \downarrow \mathbf{H P}^{\infty}$ le fibré (en droites quaternioniques) universel sur le projectif quaternionique, $\zeta_{c}$ le fibré complexe sous-jacent. Si $\Phi: \mathbf{C P}^{\infty} \rightarrow$ $\operatorname{HP}^{\infty}$ est la fibration canonique alors $\Phi^{*}\left(\zeta_{c}\right)=\xi+\bar{\xi}$ oì $\xi$ est le fibré (en droites complexes) universel sur le projectif complexe.

Maintenant $\zeta \widehat{\otimes} \zeta=\zeta_{c} \hat{\otimes} \zeta_{c}$ donc $\Phi^{*}(\zeta \hat{\otimes} \zeta)=\Phi^{*}\left(\zeta_{c}\right) \hat{\hat{\otimes}} \Phi^{*}\left(\zeta_{c}\right)=(\eta+\bar{\eta}) \otimes$ $\left(\xi+\bar{\xi}\right.$ ) (ici $\eta$ est une autre copie du fibré complexe universel sur $\mathbf{C P} \mathbf{P}^{\infty}$ ). Dans ce qui suit on omettera d'écrire le symbole $\bar{x}$.

$$
\Phi^{*}(\zeta \widehat{\otimes} \zeta)=\eta \xi+\bar{\eta} \bar{\xi}+\eta \bar{\xi}+\bar{\eta} \xi
$$

$\Phi^{*}(\zeta \otimes \zeta)$ étant un fibré complexe de rang 4 , on a

$$
\left\{\begin{array}{l}
c_{2}^{h}\left(\Phi^{*}(\zeta \widehat{\otimes} \zeta)\right)=c_{2}^{h}(\eta \xi+\bar{\eta} \bar{\xi})+c_{2}^{h}(\eta \bar{\xi}+\bar{\eta} \xi)+c_{1}^{h}(\eta \xi+\bar{\eta} \bar{\xi}) c_{1}^{h}(\eta \bar{\xi}+\eta \bar{\xi}) \\
c_{2}^{h}(\eta \xi+\bar{\eta} \bar{\xi})=F(u, v) F(\bar{u}, \bar{v})=g^{-1}(g(u)+g(v)) g^{-1}(-g(u)-g(v))
\end{array}\right.
$$

Soit $B$ une série formelle à coefficients dans $h^{*}(p t) \otimes \mathbf{Q}$ dfinie $\operatorname{par}(\mathrm{cf}$. [2])

$$
B^{-1}\left(-X^{2}\right)=g^{-1}(X) g^{-1}(-X),
$$

alors $B^{-1}\left(-g(X)^{2}\right)=g^{-1}(g(X)) g^{-1}(-g(X))=X \bar{X}$ et $g(X)^{2}=-B(X \bar{X})$. De plus $g(X)=X+\left(X^{2}\right) \Rightarrow B(X)=X+\left(X^{2}\right)$. Donc

$$
\begin{aligned}
c_{2}^{h}(\eta \xi+\bar{\eta} \bar{\xi}) & =B^{-1}\left(-(g(u)+g(v))^{2}\right) \\
& =\Phi^{*} B^{-1}\left((\sqrt{B(x)}-\sqrt{B(y)})^{2}\right)=\Phi^{*}\left(F^{-}\right)
\end{aligned}
$$


On a noté ici $\Phi^{*}(x)=u \bar{u}$ et $\Phi^{*}(y)=v \bar{v}$ où $u$ et $v$ sot les classes de Thom de $\eta$ et $\xi$ respectivement.

$$
\begin{aligned}
c_{2}^{h}(\bar{\eta} \xi+\eta \bar{\xi}) & =F(\bar{u}, v) F(u, \bar{v})=g^{-1}(-g(u)+g(v)) g^{-1}(g(u)-g(v)) \\
& =B^{-1}\left(-(g(u)-g(v))^{2}\right) \\
& =\Phi^{*} B^{-1}\left((\sqrt{B(x)}+\sqrt{B(y)})^{2}\right)=\Phi^{*}\left(F^{+}\right)
\end{aligned}
$$

Alors

$$
\Phi^{*}\left(F^{+}+F^{-}\right)=\Phi^{*}\left[c_{2}^{h}(\zeta \widehat{\otimes} \zeta)-c_{1}^{h}(\eta \xi+\eta \xi) c_{1}^{h}(\bar{\eta} \xi+\eta \bar{\xi})\right.
$$

Maintenant, il est facile de voir que

$$
\Phi^{*}\left(F^{+} \cdot F^{-}\right)=\Phi^{*}\left(c_{4}^{h}(\zeta \widehat{\otimes} \zeta)\right) .
$$

Proposition 3.1.1. Le bigroupe formel $\mathcal{F}^{2}-\left(F^{+}+F^{-}\right) \mathcal{F}+F^{+} \cdot F^{-}$est

1. principal du $1^{e r}$ type

2. à coefficients dans $h^{*}(p t)$

\section{Démonstration :}

1. est vrai d'après la proposition 2.2.5.

2. est vrai car en posant $\theta_{1}(x, y)=F^{+}+F^{-}$et $\theta_{2}(x, y)=F^{+} \cdot F^{-}$on obtient

$$
\left\{\begin{aligned}
\left(\Phi^{*} \times \Phi^{*}\right) \theta_{1}\left(c_{1}^{h}\left(\zeta_{c}\right) \otimes 1,1 \otimes c_{1}^{h}\left(\zeta_{c}\right)\right)= & \left(\Phi^{*} \times \Phi^{*}\right) c_{2}^{h}\left(\zeta_{c} \widehat{\otimes} \zeta_{c}\right) \\
& -c_{1}^{h}(\xi \widehat{\otimes} \xi+\bar{\xi} \hat{\otimes} \bar{\xi}) c_{1}^{h}(\xi \hat{\otimes} \bar{\xi}+\bar{\xi} \hat{\otimes} \xi) \\
\theta_{2}\left(c_{2}^{h}\left(\zeta_{c}\right) \otimes 1,1 \otimes c_{2}^{h}\left(\zeta_{c}\right)\right)= & c_{4}^{h}\left(\zeta_{c} \hat{\otimes} \zeta_{c}\right)
\end{aligned}\right.
$$

Remarque 3.1.2. : si $h^{*}$ est une théorie spéciale (i.e. $c_{1}^{h}(\bar{\eta})=-c_{1}^{h}(\eta)$ pour tout fibré complexe $\eta$ ), alors :

$$
\begin{aligned}
& \theta_{1}\left(c_{1}^{h}\left(\zeta_{c}\right) \otimes 1,1 \otimes c_{1}^{h}\left(\zeta_{c}\right)\right)=c_{2}^{h}\left(\zeta_{c} \widehat{\otimes} \zeta_{c}\right) \\
& \theta_{2}\left(c_{2}^{h}\left(\zeta_{c}\right) \otimes 1,1 \otimes c_{2}^{h}\left(\zeta_{c}\right)\right)=c_{4}^{h}\left(\zeta_{c} \widehat{\otimes} \zeta_{c}\right)
\end{aligned}
$$

\section{Exemples 3.1.3}


a) $h^{*}=H^{*}$ la cohomologie ordinaire,

son groupe formel est le groupe additif $F_{a}(X, Y)=X+Y$; son $\log$ arithme est la série $g(X)=X$, donc $B(X)=X$. Alors le bigroupe formel est $\mathcal{F}_{a}$.

b) $h^{*}=K$-théorie,

son groupe formel est le groupe multiplicatif $F_{m}(X, Y)=X+Y-$ $\beta X . Y$, son logarithme est la série formelle $g(X)=-\frac{1}{\beta} \log (1-\beta X)$. Alors son bigroupe formel associé est,

$$
\mathcal{F}_{m}^{2}-\left(2(X+Y)-\beta^{2} X Y\right) \mathcal{F}_{m}+(X-Y)^{2}=0,
$$

et son logarithme est

$$
B(X)=\left(\int_{0}^{\sqrt{X}} \frac{d t}{\sqrt{1-(\beta t / 2)^{2}}}\right)^{2}
$$

c) $h^{*}=$ la cohomologie elliptique,

son groupe formel est le groupe d'Euler

$$
F(X, Y)=\frac{X \sqrt{R(Y)}+Y \sqrt{R(X)}}{1-\epsilon X^{2} Y^{2}}
$$

son logarithme est la série formelle

$$
g(X)=\int_{0}^{X} \frac{d t}{\sqrt{R(t)}}
$$

ò $R(X)=1-2 \delta X^{2}+\epsilon X^{4}$ (cf. [5]).

Le bigroupe formel associé est

$$
\mathcal{F}_{e}^{2}-\frac{2(X+Y)+2 X Y(4 \delta+\epsilon(X+Y))}{(1-\epsilon X Y)^{2}}+\mathcal{F}_{e} \frac{(X-Y)^{2}}{(1-\epsilon X Y)^{2}}=0
$$

son logarithme est la série

$$
B(X)=\left(\int_{0}^{\sqrt{X}} \frac{d t}{\sqrt{1+2 \delta t^{2}+\epsilon t^{4}}}\right)^{2} .
$$




\subsection{Le cas quaternionique}

Soit maintenant $E$ un spectre-anneau définissant une théorie cohomologique C-orientée spéciale. $E^{*}\left(\mathbf{H P}^{\infty}\right)=E^{*}(p t)[[x]]$ (cf. [9]) ò $x \in E^{4}\left(\mathbf{H P}^{\infty}\right)$ est la classe de Thom de $\zeta_{c}$ (cf. section 3$)$. Par conséquent $E_{*}\left(\mathbf{H P}^{\infty}\right)$ est un $E_{*}(p t)$-module libre de base $\left\{\beta_{n}^{E}\right\}_{n \in \mathrm{N}}, \beta_{n}^{E} \in E_{\mathbf{4} n}\left(\mathbf{H P}^{\infty}\right)$, duale du système $\left\{x^{n}\right\}_{n \in \mathrm{N}}$ par le produit de Kronecker.

Soit $H_{m}:\left(\mathbf{H P}^{\infty} \times \mathbf{H} \mathbf{P}^{\infty}\right)^{+} \rightarrow \mathbf{H P}^{\infty+}$ l'application du transfert stable définie par la fibration $\Phi: \mathbf{C P}^{\infty} \rightarrow \mathbf{H P}^{\infty}$ (cf. [1] ). Par passage à l'homologie, ${ }^{H} m_{*}$ confère à $E_{*}\left(\mathbf{H} \mathbf{P}^{\infty}\right)$ une structure d'anneau.

D'autre part, dans le cas de la cohomologie ordinaire $H^{*}(-, \mathbf{Z}), H_{m^{*}}=$ $4 \Delta_{a}$ où $\Delta_{a}$ est le coproduit sur $\mathbf{Z}\left[\frac{\mathbf{1}}{\mathbf{2}}\right][[\mathbf{X}]]$ dfini par le $\mathcal{F}_{a}$ (cf.[1]), donc le produit dans $H_{*}\left(\mathbf{H P} \mathbf{P}^{\infty}, \mathbf{Z}\right)$ est donné par

$$
\begin{aligned}
\beta_{i}^{H} \beta_{j}^{H} & =H_{m *}:\left(\beta_{i}^{H} \otimes \beta_{j}^{H}\right)=\sum_{k}\left\langle x^{k}, H_{m *}:\left(\beta_{i}^{H} \otimes \beta_{j}^{H}\right)\right\rangle \beta_{k}^{H} \\
& =\sum_{k} H_{m *}\left\langle x^{k},\left(\beta_{i}^{H} \otimes \beta_{j}^{H}\right)\right\rangle \beta_{k}^{H}=4 \sum_{k}\left\langle\Delta_{a}\left(x^{k}\right),\left(\beta_{i}^{H} \otimes \beta_{j}^{H}\right)\right\rangle \beta_{k}^{H} \\
& =4 \sum_{k} \sum_{t=0}^{k} C_{2 t}^{2 k}\left\langle x^{t} \otimes x^{k-t},\left(\beta_{i}^{H} \otimes \beta_{j}^{H}\right)\right\rangle \beta_{k}^{H} \\
& =4 C_{2 i}^{2 i+2 j} \beta_{i+j}^{H} .
\end{aligned}
$$

Alors

$$
\left(\beta_{1}^{H}\right)^{n}=4^{n-1} \frac{(2 n) !}{2^{n}} \beta_{n}^{H}
$$

d'où

$$
\beta_{n}^{H}=4 \frac{2^{n}}{(2 n) !} p_{n}\left(\beta_{1}^{H}\right)
$$

où $p_{n}$ est le $n^{\text {ème }}$ polynôme de la suite binomiale associée à la $\delta$-fonctionnelle $4 \mathcal{A} ; \mathcal{A}$ etant la $\delta$-fonctionnelle canonique (i.e. $\left.\left\langle\mathcal{A}^{j}, X^{i}\right\rangle=\frac{(2 i) !}{2^{i}} \delta_{i, j}\right)$. De plus $H_{*}\left(\mathbf{H} \mathbf{P}^{\infty}, \mathbf{Q}\right)=\mathbf{Q}\left[\left[\beta_{1}^{\mathbf{H}}\right]\right]$.

Maintenant on est en mesure d'noncer le rsultat principal de ce papier:

Théorème 3.2.1. Soient $E$ un spectre-anneau définissant une théorie cohomologique C-orientée spéciale $E^{*}$ et $B \in E^{*}(p t) \otimes \mathbf{Q}[[\mathbf{X}]]$ le logarithme de son bigroupe formel. Si $\tau_{*}: E_{*}\left(\mathbf{H} \mathbf{P}^{\infty}\right) \rightarrow E^{*}(p t) \otimes \mathbf{Q}\left[\left[\beta_{1}^{\mathbf{H}}\right]\right]$ est l'homomorphisme d'Hurewicz alors, 


$$
\tau_{*}\left(\beta_{n}^{E}\right)=4 \frac{2^{n}}{(2 n) !} q_{n}\left(\frac{\beta_{1}^{H}}{4}\right)
$$

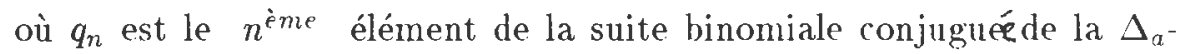
fonctionnelle d'indicatric $\epsilon B$, relativement an calcul "umbral" défini par le b.f.e.1. $\operatorname{sur} E^{*}(p t) \otimes \mathbf{Q}[[\mathbf{X}]]$.

Démonstration : $E^{*}$ est une théorie cohomologique $\mathbf{C}$-orientée spéciale , donc le logarithme $g$ de son groupe formel est une série formelle impaire liée à $B$ par la relation $B\left(-X^{2}\right)=-g(X)^{2}$. En identifiant les classes de Thom du fibré universel $\xi\rfloor \mathbf{C} \mathbf{P}^{\infty} u^{E}, u^{H}$ relativement à $E$ et ' $\mathcal{Q} \mathbf{Q}$ à leurs images dans $E \wedge \mathcal{H Q}$ on a $u^{H}=g\left(u^{E}\right)$; et en faisant de même pour les classes de Thom du fibré $\zeta_{c} \downarrow \mathbf{H} \mathbf{P}^{\infty}, x^{E}, x^{H}$ on obtient $\Phi^{*}\left(x^{H}\right)=-\left(u^{E}\right)^{2}$. Donc $x^{H}=B\left(x^{E}\right)$. Par dualité nous déduisons $\beta_{i}^{E}=\sum_{i} d_{i}^{j} \beta_{j}^{H}$ où les $d_{i}^{j}$ sont donnés par $B(X)^{j}=\sum_{i} d_{i}^{j} X^{i}$

En interprétant $B(X)$ comme l'indicatrice d'une $\Delta$-fonctionnelle à coefficients dans $E^{*}(p t) \otimes \mathbf{Q}$ muni du calcul "umbral" induit par le b.f.e.1. on obtient $d_{i}^{j}=\left\langle B(\mathcal{A})^{j}, \frac{2^{i}}{(2 i) !} X^{i}\right\rangle$.

De plus on a $\left.\left(\beta_{1}^{H}\right)^{n}=4^{n-1} \frac{(2 n) !}{2^{n}} \beta_{n}^{H}\right)$, donc

$$
\begin{aligned}
\beta_{i}^{E} & =\sum_{j}\left\langle B(\mathcal{A})^{j}, \frac{2^{i}}{(2 i) !} X^{i}\right\rangle \beta_{j}^{H}=4 \sum_{j}\left\langle B(\mathcal{A})^{j}, \frac{2^{i}}{(2 i) !} X^{i}\right\rangle \frac{2^{j}}{(2 j) !}\left(\frac{\beta_{1}^{H}}{4}\right)^{j} \\
& =4 \frac{2^{i}}{(2 i) !} \sum_{j}\left\langle B(\mathcal{A})^{j}, X^{i}\right\rangle \frac{2^{j}}{(2 j) !}\left(\frac{\beta_{1}^{H}}{4}\right)^{j}=4 \frac{2^{i}}{(2 i) !} q_{i}\left(\frac{\beta_{1}^{H}}{4}\right)
\end{aligned}
$$

Remarque 3.2.2., Par souci de simplicité on a identifié les $\beta_{j}^{H}$ dans $H_{*}\left(\mathbf{H} \mathbf{P}^{\infty}, \mathbf{Q}\right)$ avec leurs images par l'homomorphisme d'Hurewicz dans ( $E \wedge$ $\mathcal{H Q})_{*}\left(\mathbf{H} \mathbf{P}^{\infty}\right)$.

\section{Exemples 3.2.3.}

1) Le cas de la cohomologie ordinaire a été traité au début de cette section.

2) Cas de la cohomologie elliptique :

Notons $E l l$ le spectre-anneau représentant cette théorie (cf. exemple 3.1.3 c) ci-dessus). $E l_{*}\left(\mathbf{H P}^{\infty}\right)=E l_{*}(p t)\left\{\beta_{j}^{E l}\right\}$ o $E l l_{*}(p t)=\mathbf{Z}\left[\frac{1}{2}\right]\left[\delta, \epsilon, \Delta^{-1}\right]$ et $\Delta=\epsilon\left(\delta^{2}-\epsilon\right)^{2}$. 
Si $\tau_{*}: E l l_{*}\left(\mathbf{H} \mathbf{P}^{\infty}\right) \rightarrow(E l l \wedge \mathcal{H} \mathbf{Q})_{*}\left(\mathbf{H} \mathbf{P}^{\infty}\right)$ est l'homomorphisme d'Hurewicz, alors :

$$
\tau_{*}\left(\beta_{n}^{E l l}\right)=4 \frac{2^{n}}{(2 n) !} \sum_{j}\left\langle B(\mathcal{A})^{j}, X^{n}\right\rangle \frac{2^{j}}{(2 \underline{2}) !}\left(\frac{\beta_{1}^{H}}{4}\right)^{j}
$$

oì

$$
\begin{aligned}
B(X) & =\left(\int_{0}^{\sqrt{X}} \frac{d t}{\sqrt{1+2 \delta t^{2}+\epsilon t^{4}}}\right)^{2}=X\left(\sum_{n}(-1)^{n} \frac{P_{n}(\delta, \epsilon)}{2 n+1} X^{n}\right)^{2} \\
& =\sum_{n \geq 0}(-1)^{n} \sum_{a+b=n} \frac{P_{a}(\delta, \epsilon)}{2 a+1} \frac{P_{b}(\delta, \epsilon)}{2 b+1} X^{n+1} .
\end{aligned}
$$

oì $P_{n}(\delta, \epsilon):=P_{n}\left(\frac{\delta}{\sqrt{\epsilon}}\right)$ avec $P_{n}$ est le $n^{\text {ème }}$ polynôme de Legendre.

Alors on a :

$$
\tau_{*}\left(\beta_{n}^{E l l}\right)=(-1)^{n} \sum_{j \grave{ } 0}(-1)^{j} \sum_{\mathrm{i} j=\mathrm{n}}\left\{\prod_{k=1}^{j} \sum_{a+b=i_{k}-1} \frac{P_{a}(\delta, \epsilon)}{2 a+1} \frac{P_{b}(\delta, \epsilon)}{2 b+1}\right\} \frac{\left(\beta_{1}^{H}\right)^{j}}{2^{j-2}(2 j) !} .
$$

\section{Références :}

[1] V.M. BUKHSHTABER, Characteristic cobordism classes and topological application of the theory of one valued and two valued formal groups. Itogi Nauki i Techniki : Sovremenny Problemy Mat., Vol. 10, VINITI, Moscow, 1978, pp. 5-178; English transl.in J.Soviet Math. 11 , No. 6 (1979).

[2] V.M. BUKHSHTABER and P.S. NOVIKOV, Formal groups, power systems, and Adams operators. Mat.Sb. 84 (126), pp. $81-118$; English transl.in Math. USSR Sb.13 (1979).

[3] A.FROHLICH, Formal groups. Lecture Notes in Mathematics, 74, Springer-Verlag, (1968).

[4] A.N.KHOLODOV, The umbral calculus on multivalued formal groups, and Adams projections in K-theory. Math.USSR Sbornik,Vol. 65, No. $2(1990)$.

[5] P.S.LANDWEBER, Elliptic cohomology and modular forms. Lecture Notes in Mathematics, 1326, Springer-Verlag. 
[6] N.RAY, Extensions of umbral calculus : pneumbral conlgebras and generalised Bernoulli numbers. Advances in Math. 61, pp. 49-100 (1986).

[7] N.RAY, 'Symbolic calculus : A 19th century approch to MU and BP', in 'Homotopy theory'. J. D. S. Jones \& E. Rees (erds), LMS Lect. Notes Ser. 117, CUP, pp. $195-238$ (1987).

[8] S.ROMAN and G-C. ROTA, The umbral calculus. Advances in Math. 27 , pp. $95-188$ (1978).

[9] R.E.STONG, Notes on cobordism theory. Princeton University Press, N.J., and Univ.Tokyo Press,Tokyo,(1968).

[10] R.M.SWITZER, Algebraic topology-homotopy and homology. Springer-Verlag, (1975).

Received : October 15, 1995.

\section{Fayçal Lamrini}

Département de Mathématique et Informatique

Faculté des Sciences Dhar-Mehraz

Université Sidi Mohammed Benabdallah

B. P. 1796, FES (MAROC)

Marruecos 